\title{
Prompt production of loosely bound mesonic molecules at hadron colliders
}

\section{Christopher Bignamini}

Dipartimento di Fisica Nucleare e Teorica, Università di Pavia and INFN Sezione di Pavia, Via A. Bassi 6, I-27100 Pavia, Italy

Institut für Theoretische Physik, Universität Karlsruhe, D-76128 Karlsruhe, Germany

E-mail: christopher.bignamini@pv.infn.it

\section{Benjamin Grinstein}

CERN-PH-TH, CH-1211 Geneva 23, Switzerland

University of California, San Diego, Department of Physics, La Jolla, CA 92093-0315, USA

E-mail: bgrinstein@ucsd.edu

\section{Fulvio Piccinini}

INFN Sezione di Pavia, Via A. Bassi 6, I-27100 Pavia, Italy

E-mail: fulvio.piccinini@pv.infn.it

\section{Antonio Davide Polosa}

INFN Roma, Piazzale A. Moro 2, Roma, I-00185, Italy

E-mail: antonio.polosa@romal.infn.it

\section{Chiara Sabelli*}

Università di Roma La Sapienza and INFN Roma, Piazzale A. Moro 2, Roma, I-00185, Italy

E-mail: chiara.sabellieromal.infn.it

\begin{abstract}
Many exotic hadron candidates have been identified among the XYZ particles observed at the $B$-factories and in $p \bar{p}$ collisions at Tevatron since 2003. Among these resonances, the first to be discovered was the $X(3872)$, which has been identified by many authors with a $D$-mesons molecule. We test this hypothesis on the prompt production cross section as it is measured by CDF. The result of this test seems to challenge the molecular interpretation of this state.
\end{abstract}

XVIII International Workshop on Deep-Inelastic Scattering and Related Subjects April 19 -23, 2010

Convitto della Calza, Firenze, Italy

\footnotetext{
* Speaker.
} 


\section{Introduction}

With the discovery of the first charmonium state, the $J / \psi$, in 1974 hadronic spectroscopic entered in a new era, as $c \bar{c}$ bound states offered the possibility to study strong interactions using a non relativistic approach. While the predictions for states below the open charm threshold were confirmed by experiments, the scan above that threshold uncovered many new resonances decaying into low-lying charmonia but which could not be identified with standard $c \bar{c}$. These evidences lead many authors to reconsider the several exotic possibilities proposed for hadrons at the very beginning of QCD. Among them there are the multiquark states: meson-meson molecules, bound states of two mesons, and tetraquarks, bound states of a diquark and an antidiquark. The first possibility has been widely explored for the case of $X(3872)$, as we will review in Sec. 2. We will discuss the implication of this hypothesis on the prompt production cross section in Sec. 3. In Sec. 4 the role of Final State Interactions in the formation of molecular bound states will be considered. Finally, in Sec. 5 the tetraquark alternative will be briefly described.

\section{The molecular hypothesis}

$X$ (3872) was first observed in August 2003 by Belle [1] in $B$-meson decays, in the $J / \psi \pi^{+} \pi^{-}$ invariant mass distribution at about $3872 \mathrm{MeV}$. Soon after it was confirmed by BaBar [2] and in $p \bar{p}$ collisions at the Tevatron by CDF and D0 [3]. The decay width of this resonance appears smaller than the experimental resolution of $\sim 2.3 \mathrm{MeV}$. The $J^{P C}$ assignation is still controversial [4]: in what follows we will assume $J^{P C}=1^{++}$.

The possibility to identify the $X$ with a standard $2^{3} P_{1}$ charmonium state was not straightforward mainly because of its extremely small decay width and of the ratio between radiative and hadronic branching fractions [5]. The vicinity of the mass of the $X$ to the $D^{0} \bar{D}^{0 *}$ threshold thus suggested to identify the $X$ with a $D^{0} \bar{D}^{0 *} S$-wave molecule at threshold [6]. The wave function is

$$
\frac{\left|D^{0} \bar{D}^{0 *}\right\rangle+\left|\bar{D}^{0} D^{0 *}\right\rangle}{\sqrt{2}}
$$

This hypothesis was reinforced when it was established that the $X$ decays to $J / \psi \rho$ and $J / \psi \omega$ with the same strength [7]. The light flavor content of the $D^{0(*)}$ mesons can account for the maximal isospin violation observed.

The most striking characteristic of this meson-meson bound state would be its tiny binding energy $\varepsilon_{0}=(-0.25 \pm 0.4) \mathrm{MeV}$, namely compatible with zero. A small binding energy means a large scattering length and thus an extremely non compact state. It seems at odds with common intuition that such a loosely bound state could be promptly produced with a consistent amount in high energy proton anti-proton collisions, and thus it is worth to focus on the prompt production cross section of the $X$ in $p \bar{p}$ collisions.

\section{Prompt Production Cross Section}

In a recent paper [8] we tested the molecular assignation against the prompt production mechanism. A recent analysis by CDF [9] allows to distinguish the fraction of $X$ and $\psi(2 S)$ produced 
promptly from the one originated from $B$-decays. The result of this study, which used nearly identical selection criteria for the two states, reported a prompt produced fraction of $(83.9 \pm 5.3) \%$ and $(71.7 \pm 1.2) \%$ for $X$ and $\psi(2 S)$ respectively. Assuming the same rapidity distribution for $X$ and $\psi(2 S)$ in the range $|y|<1$ and using the estimate of the prompt production cross section of $\psi(2 S)$ at $\mathrm{CDF}$ contained in [10], one can obtain the following product:

$$
\sigma(p \bar{p} \rightarrow X(3872)+\text { all })_{\text {prompt }} \times \mathscr{B}\left(X(3872) \rightarrow J / \psi \pi^{+} \pi^{-}\right)=(3.1 \pm 0.7) \mathrm{nb}
$$

for $p_{T} \geq 5 \mathrm{GeV}|y| \leq 0.6$. Using some bounds on the branching fraction [11] one obtains $33 \mathrm{nb}<$ $\sigma(p \bar{p} \rightarrow X(3872)+\text { all })_{\text {prompt }}<72 \mathrm{nb}$. To estimate an upper bound for the theoretical prompt production cross section we made use of a Schwartz inequality:

$$
\begin{aligned}
& \sigma(p \bar{p} \rightarrow X(3872)) \sim\left|\int d^{3} \boldsymbol{k}\left\langle X \mid D \bar{D}^{*}(\boldsymbol{k})\right\rangle\left\langle D \bar{D}^{*}(\boldsymbol{k}) \mid p \bar{p}\right\rangle\right|^{2} \simeq\left|\int_{\mathscr{R}} d^{3} \boldsymbol{k}\left\langle X \mid D \bar{D}^{*}(\boldsymbol{k})\right\rangle\left\langle D \bar{D}^{*}(\boldsymbol{k}) \mid p \bar{p}\right\rangle\right|^{2} \\
& \leq \int_{\mathscr{R}} d^{3} \boldsymbol{k}|\psi(\boldsymbol{k})|^{2} \int_{\mathscr{R}} d^{3} \boldsymbol{k}\left|\left\langle D \bar{D}^{*}(\boldsymbol{k}) \mid p \bar{p}\right\rangle\right|^{2} \leq \int_{\mathscr{R}} d^{3} \boldsymbol{k}\left|\left\langle D \bar{D}^{*}(\boldsymbol{k}) \mid p \bar{p}\right\rangle\right|^{2} \sim \sigma(p \bar{p} \rightarrow X(3872))^{\max }
\end{aligned}
$$

where $k$ is the relative 3-momentum between the $D$ mesons in the center of mass frame of the pair, $\psi(\boldsymbol{k})$ is some bound state wave function, and $\mathscr{R}$ is the region were the wave function is appreciably different from zero. To estimate the size of $\mathscr{R}$ we use standard quantum mechanics. Assuming a gaussian wave function and relying on one pion exchange to obtain the binding between the mesons, we can deduce the size of the molecule to be $r_{0} \simeq 8 \mathrm{fm}$. Furthermore, using a minimal uncertainty principle we obtain the spread of the relative 3-momentum $\Delta k \sim \hbar / 2 r_{0} \simeq 12 \mathrm{MeV}$ around a central value which can be computed from the decay momentum $k_{0}=\sqrt{\lambda\left(m_{x}^{2}, m_{D}^{2}, m_{D *}^{2}\right)} / 2 m_{x} \simeq 27 \mathrm{MeV}$. Thus $\mathscr{R}=\left[0, k_{0}+\Delta k\right] \simeq[0,35] \mathrm{MeV}$. We recover what we expected: the two mesons inside the molecule must be almost collinear to account for such a small binding energy.

The matrix element $\left\langle D \bar{D}^{*}(\boldsymbol{k}) \mid p \bar{p}\right\rangle$ can be computed using standard hadronization Monte Carlo programs like Herwig and Pythia. To do so, we required our MC tools to generate $2 \rightarrow 2 \mathrm{QCD}$ events with some loose partonic cuts. In the hadron samples produced by the hadronization algorithm we list the events containing $D^{0} \bar{D}^{0 *}$ as a function of their center of mass relative 3 -momentum. We tune our MC tools on CDF data on $D^{0} D^{*-}$ pair production. In Fig. 1 we report the differential distribution for the upper bound on the prompt production.

Integrating the distribution up to $k_{\max } \sim 35 \mathrm{MeV}$ we obtain $\sigma_{\text {prompt }}^{\text {th }} \leq 0.075 \mathrm{nb}$ with Herwig, and $\sigma_{\text {prompt }}^{\text {th }} \leq 0.11 \mathrm{nb}$ with Pythia, which are both about 300 times smaller than the lower limit on the experimental prompt production cross section measured at CDF. This result challenged the molecular interpretation of the $X$. Nevertheless soon after it was claimed that the effect of final state interactions between the $D$ mesons was sufficient to reconcile theory and experiment. We discuss this issue in the next section.

\section{The role of Final State Interactions}

In a subsequent paper by Braaten and Artoisenet [12] the effect of final state interactions (FSI) between the $D^{0}$ and $\bar{D}^{0 *}$ mesons has been taken into account: FSI should be able to rescatter 

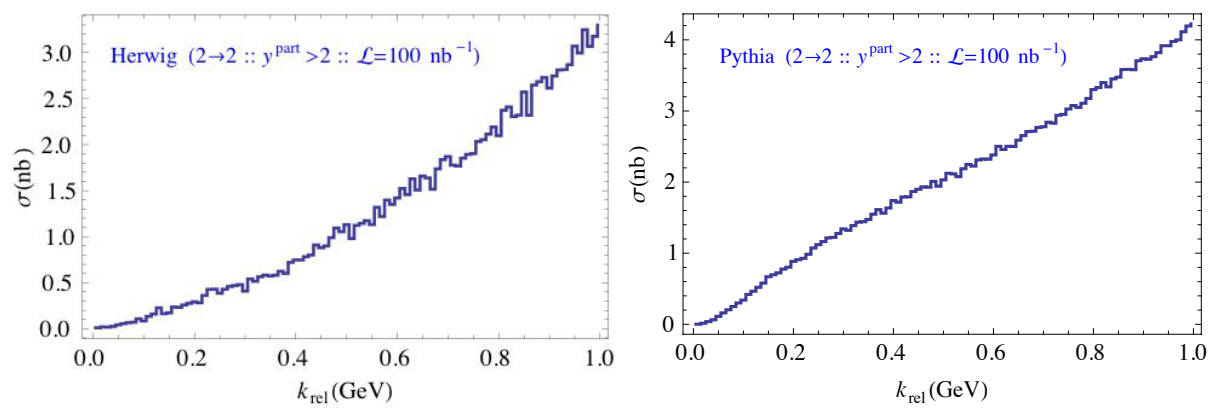

Figure 1: Left: the integrated cross section obtained with Herwig as a function of the relative momentum of the mesons in the $D^{0} \bar{D}^{0 *}$ molecule. This plot is obtained after the generation of $55 \times 10^{9}$ events with parton cuts $p_{\perp}^{\text {part }}>2 \mathrm{GeV}$ and $y^{\text {part }}>6$. The cuts on the final $D$ mesons are such that the molecule produced has a $p_{\perp}>5 \mathrm{GeV}$ and $y<0.6$. Right: same plot but using Pythia.

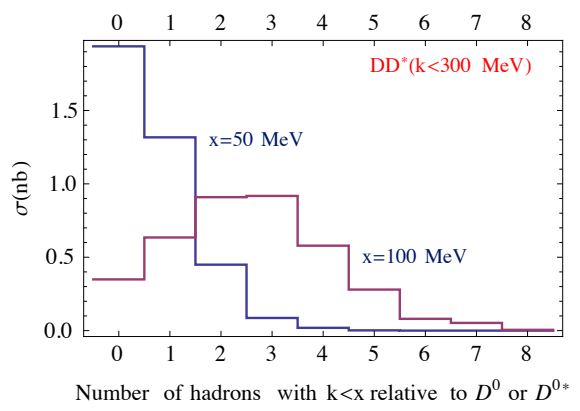

Figure 2: The cross section integrated in bins containing $n=0,1,2, \ldots$ extra hadrons having a relative momentum $k<x \mathrm{MeV}$ with respect to the $D$ or the $D^{*}$ composing the $X(3872)$ molecule. Following [12] it is assumed that the molecule is formed in $S$-wave with a relative $k$ in the center of mass of $D$ and $D^{*}$ as large as $300 \mathrm{MeV}$.

higher relative momentum pairs to lower relative momentum pairs, thus allowing to consider $k_{\max } \sim$ $2 m_{\pi}$ and thus to integrate a larger cross-section. Furthermore the authors of [12] introduce an enhancement factor in the cross-section $\sim 1 /\left(k^{2}+r_{0}^{2}\right)$, as stated in the Migdal-Watson theorem. In this way they succeed in reconciling the theoretical and experimental values.

However in [13] we questioned about the possibility to quantify the enhancement caused by FSI using the Migdal-Watson theorem, due to the presence of extra-hadrons close to the $D$ mesons in 3-momentum space in the final state. There are two main conditions that have to be met in order to safely apply the theorem. First, the relative momentum between the two rescattering particles has to be smaller than the inverse of the range of strong interactions, namely $200 \mathrm{MeV}$. Second, it is necessary that no more than two hadrons match the first condition. Reference [13] showed that the latter is not satisfied in the MC samples obtained in [8]. Indeed Fig. 2 shows that the average number of additional hadrons with relative 3-momentum smaller than $x=50$ and $100 \mathrm{MeV}$ with respect to the one of the two $D$ mesons which are candidates to form a $X$, is 2 and 3 respectively.

Furthermore going up to relative momentum as large as $2 m_{\pi}$ one cannot ignore higher partial wave scattering beside the $S$-wave one. The debate remains open. 


\section{The tetraquark alternative}

The main alternative in the interpretation of the $X(3872)$ was proposed by Maiani et al. in [14], where the $X$ was comprehended as a compact tetraquark state, namely a diquark-antidiquark $S$ wave bound state: $X \sim[c q]_{0}[\bar{c} \bar{q}]_{1}+[c q]_{1}[\bar{c} \bar{q}]_{0}$. The different color structure implies a different fragmentation process which may account for the large prompt production cross section, even if any quantitative estimate is not possible at present times. The main drawback of the tetraquark option remains the prediction of two different neutral states with masses within few $\mathrm{MeV}$, which has not been established even if some evidence is still reported [15], and of two charged states in the $3.8 \mathrm{GeV}$ region, which have never been observed.

\section{References}

[1] S. K. Choi et al. [Belle Collaboration], Phys. Rev. Lett. 91, 262001 (2003).

[2] B. Aubert et al. [BABAR Collaboration], Phys. Rev. D 71, 071103 (2005).

[3] D. E. Acosta et al. [CDF II Collaboration], Phys. Rev. Lett. 93, 072001 (2004). V. M. Abazov et al. [D0 Collaboration], Phys. Rev. Lett. 93, 162002 (2004).

[4] K. Abe et al. [Belle Collaboration], arXiv:hep-ex/0505038; A. Abulencia et al. [CDF Collaboration], Phys. Rev. Lett. 98, 132002 (2007); P. d. A. al. [BABAR Collaboration], arXiv:1005.5190 [hep-ex].

[5] T. Barnes and S. Godfrey, Phys. Rev. D 69, 054008 (2004); E. J. Eichten, K. Lane and C. Quigg, Phys. Rev. D 69, 094019 (2004);

[6] E. Braaten and M. Kusunoki, Phys. Rev. D 69, 074005 (2004); F. E. Close and P. R. Page, Phys. Lett. B 578, 119 (2004); N. A. Tornqvist, Phys. Lett. B 590, 209 (2004).

[7] K. Abe et al. [Belle Collaboration],

[8] C. Bignamini, B. Grinstein, F. Piccinini, A. D. Polosa and C. Sabelli, Phys. Rev. Lett. 103 (2009) 162001.

[9] CDF note 7159, http://www-cdf.fnal.gov/physics/new/bottom/051020.blessed-X3872/XLife/xlonglivedWWW.ps; see also A. Abulencia et al. [CDF Collaboration], Phys. Rev. Lett. 98, 132002 (2007).

[10] T. Aaltonen et al. [CDF Collaboration], Phys. Rev. D 80, 031103 (2009).

[11] B. Aubert et al. [BABAR Collaboration], Phys. Rev. Lett. 96, 052002 (2006).

[12] P. Artoisenet and E. Braaten, Phys. Rev. D 81, 114018 (2010).

[13] C. Bignamini, B. Grinstein, F. Piccinini, A. D. Polosa, V. Riquer and C. Sabelli, Phys. Lett. B 684, 228 (2010).

[14] L. Maiani, F. Piccinini, A. D. Polosa and V. Riquer, Phys. Rev. D 71, 014028 (2005).

[15] B. Aubert et al. [BABAR Collaboration], Phys. Rev. D 77 (2008) 111101;

B. Aubert et al. [BABAR Collaboration], Phys. Rev. D 77, 011102 (2008);

I. Adachi et al. [Belle Collaboration], arXiv:0809.1224 [hep-ex];

I. Adachi et al. [Belle Collaboration], arXiv:0810.0358 [hep-ex]. 